\title{
Performance analysis of a threshold-based discrete-time queue using maximum entropy
}

\author{
L. Guan ${ }^{\text {a,* }}$, I.U. Awan ${ }^{\text {b }}$, I. Phillips ${ }^{\text {a }}$, A. Grigg ${ }^{c}$, W. Dargie ${ }^{\mathrm{d}}$ \\ ${ }^{a}$ Department of Computer Science, Loughborough University, Loughborough, LE11 3TU, UK \\ ${ }^{\mathrm{b}}$ Department of Computing, University of Bradford, Bradford, BD7 1DP, UK \\ ' System Engineering Innovation Centre, Loughborough University, Loughborough, LE11 3TU, UK \\ ${ }^{\mathrm{d}}$ Department of Computer Networks, Technical University of Dresden, Germany
}

\section{A R T I C L E I N F O}

\section{Article history:}

Available online 16 September 2008

\section{Keywords:}

Maximum entropy (ME) principle

Queue threshold

Compound Bernoulli process (CBP)

Generalised geometric (GGeo) distribution

Congestion control

\begin{abstract}
A B S T R A C T
The provision of guaranteed QoS for various Internet traffic types has become a challenging problem for researchers. New Internet applications, mostly multimedia-based, require differentiated treatments under certain QoS constraints. Due to a rapid increase in these new services, Internet routers are facing serious traffic congestion problems. This paper presents an approximate analytical performance model in a discrete-time queue, based on closed form expressions using queue threshold, to control the congestion caused by the bursty Internet traffic. The methodology of maximum entropy (ME) has been used to characterize closed form expressions for the state and blocking probabilities. A discrete-time GGeo/GGeo/1/\{ $\left.N_{1}, N_{2}\right\}$ censored queue with finite capacity, $N_{2}$, external compound Bernoulli traffic process and generalised geometric transmission times under a first come first serve (FCFS) rule and arrival first (AF) buffer management policy has been used for the solution process. To satisfy the low delay along with high throughput, a threshold, $N_{1}$, has been incorporated to slow the arrival process from mean arrival rate $\lambda_{1}$ to $\lambda_{2}$ once the instantaneous queue length has been reached, otherwise the source operates normally. This creates an implicit feedback from the queue to the arrival process. The system can be potentially used as a model for congestion control based on the Random Early Detection (RED) mechanism. Typical numerical experiments have been included to show the credibility of ME solution against simulation for various performance measures and to demonstrate the performance evaluation of the proposed analytical model.
\end{abstract}

Crown Copyright (c) 2008 Published by Elsevier B.V. All rights reserved.

\section{Introduction}

Introduction of new network services are bringing dramatic changes to the volume and nature of Internet traffic. These new services generally have different Quality of Service (QoS) requirements. These services also generate huge volume of traffic and can cause severe congestion problems leading to serious degradation of network performance in terms of high delays and packet loss. These problems pose numerous challenges to the research community for developing effective performance tools to analyze different protocols and buffer management schemes in order to guarantee the QoS for different network services. Consequently, congestion control to enable different types of Internet traffic to satisfy specified QoS constraints to accommodate the increasingly diverse range of services and types of traffic is becoming significantly more

\footnotetext{
* Corresponding author.

E-mail address: L.Guan@lboro.ac.uk (L. Guan).
} 


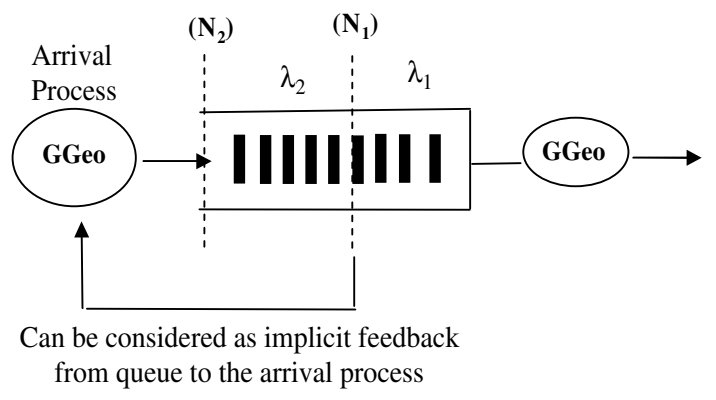

Fig. 1. GGeo/GGeo/ $1 /\left\{N_{1}, N_{2}\right\}$ censored queue.

important. Many systems in network environments require the queue to be monitored for impending congestion before it happens [1].

Congestion occurs when the aggregate demand exceeds the available capacity of resources. Performance modelling techniques help to determine the efficacy of telecommunication networks capable of providing interactive services (such as voice, data and video) by developing good models. As the volume of the Internet traffic has increased, users are likely to experience more packet loss, longer delay and other performance degradation due to congestion. One solution to this problem could be to keep the network utilization low but this may not be very cost-effective solution. Analysis of queueing systems with finite capacities can play very important roles in the performance evaluation of networks [2].

Traditionally, tail drop has been used for traffic congestion control. It sets a maximum queue capacity and drops packets when the queue becomes full, but leads to several problems including global synchronization and lock out [3]. In order to solve the problems, some active queue management ( $\mathrm{AQM}$ ) mechanisms have been proposed and implemented to manage the queue lengths, reduce end-to-end latency, reduce packet dropping, and avoid lock out phenomena so that the control of traffic congestion can be achieved by the use of appropriate buffer management schemes. These mechanisms include random early detection (RED) [4], random early marking (REM) [5,6], a virtual queue-based scheme where the virtual queue is adaptive [7-9] and a proportional integral controller mechanism [10], among others. Of the above schemes to implement $\mathrm{AQM}, \mathrm{RED}$ is the default mechanism for managing queue lengths to meet these goals in a FIFO queue, and is recommended by the Internet Society in RFC 2309 (cf [3]), Quote: "Unless a developer has reasons to provide another equivalent mechanism we recommend that RED be used".

Since RED was proposed by Floyd and Jacobson [3] in 1993, most researchers have used simulation tools as the choice of modelling approach to examine the performance of various aspects of the RED mechanism. Only a few publications, such as [11-14], have attempted to theoretically evaluate the performance of RED. To the authors' knowledge, there is no clear description of the parameter settings and exact information being measured and it is very important and necessary to use an analytical approach to address the more fundamental aspects of the RED mechanism. RED depends on setting thresholds in the queue and our research uses this principle and looks at this in a simplified way. We incorporate one threshold $N_{1}$ in the system which define changes in the arrival rate so that this system can be potentially used as a model for RED (cf Fig. 1).

This paper presents a maximum entropy (ME)-based approximate analytical solution to model the GGeo/GGeo/1/\{ $\left.N_{1}, N_{2}\right\}$ censored queue for implementing AQM. The external bursty traffic has been modelled using a compound Bernoulli process while the generalised geometric (GGeo) process represents the transmission process. A threshold $N_{1}$ has been incorporated in the discrete-time finite buffer with total capacity $N_{2}$ to control the external arrivals.

The rest of paper is organised as follows: ME methodology and GGeo-type distribution are described in Section 2. ME solution for a stable GGeo/GGeo/1/\{N,$\left.N_{2}\right\}$ censored queue with threshold $N_{1}$ and total buffer capacity $N_{2}$ is characterised in Section 3. Numerical validation results against simulation, involving generalised geometric (GGeo) inter-arrival and service time distributions together with the performance study of the proposed analytical model are included in Section 4 . Conclusions follow in Section 5.

\section{ME methodology and GGeo distribution}

\subsection{The principle of maximum entropy (PME)}

The ME methodology is an approximate technique for finding probability distributions using information theory. It is an alternative way to solve quite complex queues instead of traditional balance equations. The principle of maximum entropy (PME) $[15,16]$ provides a self-consistent method of inference for charactering an unknown but true probability distribution, subject to the mean value constraints supplied by the given information. The ME solution can be expressed in terms of a normalising constant and a product of Lagrangian coefficients corresponding to the constraints. In an Information theoretic context [15], the ME solution corresponds to the maximum disorder of system states and, thus, is considered to be the least biased distribution estimate of all solutions that satisfy the system's constraints. In sampling terms, it has been shown [16] 
that, given the imposed constraints, the ME solution can be experimentally realised in overwhelmingly more ways than any other distribution. Shore and Johnson [17] showed that the PME is a uniquely correct method of inductive inference when information is given in the form of expected values. Tribus [18] used the principle to derive a number of known probability distributions by using a variety of mean value constraints in terms of moments. More details on PME and its applications can be found in $[19,27,28]$.

\subsection{The GGeo-type distribution}

The GGeo-type distribution is an inter-arrival time or inter-departure time distribution of the form [20] (cf Fig. 2)

$$
\begin{aligned}
& f_{n}=\operatorname{Pr}(W=n)= \begin{cases}1-\tau, & n=0, \\
\tau \sigma(1-\sigma)^{n-1}, & n \geqslant 1,\end{cases} \\
& \tau=2 /\left(C^{2}+1+v\right), \quad 0 \leqslant \tau \leqslant 1, \\
& \sigma=\tau v, \quad 0 \leqslant \sigma \leqslant 1,
\end{aligned}
$$

where $W$ is a discrete-time random variable (rv) representing the inter-arrival time or inter-departure time of an individual job in a steady single sever queue, while $1 / v$ and $C^{2}$ are the corresponding mean and squared coefficient of variation (SCV) of rv $W . v$ is mean event rate (arrivals or departures per slot). The GGeo distribution is stochastically a true probability distribution when $C^{2} \geqslant|1-v|$.

The batch arrivals or departures are allowed in the GGeo-type distribution when rv $W$ achieve zero value, it is also implied that a batch inter-event pattern according to a compound Bernoulli process (CBP) with rate $\sigma$, while the number of events (e.g. arrivals or departures) in a slot (i.e. batch size) is geometrically distributed with parameter $\tau$. The GGeo pattern is generated by a sequence of batch Bernoulli independent and identically distributed non-negative integer values rv's $\left\{W_{k}\right\}$, where $W_{k}, k=1,2, \ldots$ is the number of the events occurring at the $k$ th slot, with a fixed probability distribution given by [21]

$$
g_{l}= \begin{cases}P\left(W_{k}=0\right)=1-\sigma, & l=0 \\ P\left(W_{k}=l\right)=\sigma \tau(1-\tau)^{l-1}, & l \geqslant 1\end{cases}
$$

The GGeo distribution is versatile, possessing pseudo-memoryless property which makes solutions of many GGeo-type queueing systems and networks analytically tractable. It is an extremal member of a family of two parallel-phase Geo distributions with the same mean and SCV, where one of the phase rates approaches to infinity with probability (1- $\tau$ ). More details can be found in [20,21].

The choice of the GGeo distribution is further motivated by the fact that measurements of actual traffic or service time may be generally limited and so only few parameters can be computed reliably. Typically, only the mean and variance may be relied upon. In this case, the choice of distribution which implies least biased (i.e. introduction of arbitrary and, therefore, false assumptions) within a discrete-time domain is that of GGeo-type distribution. [22]

\section{ME analysis of GGeo/GGeo/1/\{ $\left.N_{1}, N_{2}\right\}$ censored queue}

This section presents the ME analysis of a stable single server GGeo/GGeo/1/\{N,$\left.N_{2}\right\}$ censored discrete-time finite capacity queue with:

$N_{1}$ threshold value, $N_{2}$ total buffer capacity, first come first served (FCFS) scheduling discipline, arrival first (AF) buffer management policy and censored arrival process for single class jobs.

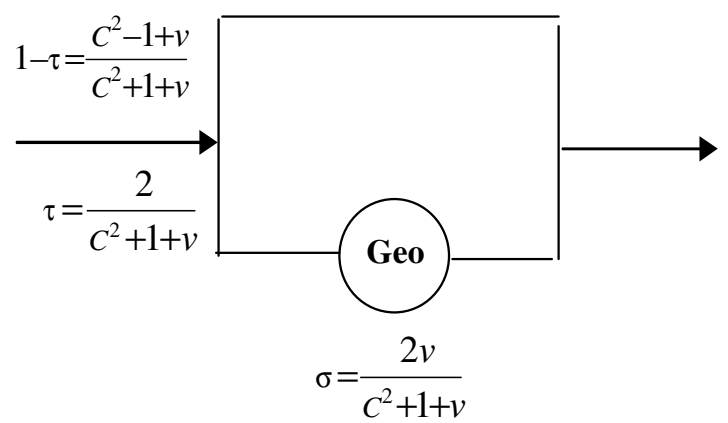

Fig. 2. The Generalised geometric (GGeo) distribution with parameters $\sigma$ and $\tau(0<\sigma, \tau \leqslant 1)$. 


\section{Notation}

Let

$S$ be the state of the queue

$Q$ be the set of all feasible states of $S$

$\Lambda$ be the arrival rate and $\Lambda=\lambda_{1}$ if instantaneous queue length reaches the threshold value $N_{1}$ or $\Lambda=\lambda_{2}$ after MQL exceed the threshold $N_{1}$

$\mu$ be the service rate

$\pi$ be the blocking probability that an arrival finds the queue full

$P(S)$ be the stationary state probability

To analyse this system, we consider two kinds of jobs in the queue; jobs that arrive with rate $\lambda_{1}(\mathrm{i}=1)$ and those that arrive after the threshold with rate $\lambda_{2}(i=2)$. For each state $S, S \in Q$ the following auxiliary functions are defined:

$N_{i}(S)=$ the number of jobs present in state $S$,

$$
\begin{aligned}
& s_{i}(S)= \begin{cases}1, & \text { if } n_{i}(S)>0, \quad i=1,2, \\
0, & \text { otherwise },\end{cases} \\
& f_{i}(S)= \begin{cases}1, & \text { if } n_{i}(S)=N_{i}, \quad i=1,2 \\
0, & \text { otherwise }\end{cases}
\end{aligned}
$$

Suppose that the following mean value constraints about the state probability $P(S)$ are known to exist:

(i) Normalisation,

$$
\sum_{S \in Q} P(S)=1
$$

(ii) Utilization,

$$
\sum_{S \in Q} s_{i}(S) P(S)=U_{i}, \quad 0<U_{i}<1, \quad i=1,2 .
$$

(iii) Mean queue length,

$$
\sum_{S \in Q} n_{i}(S) P(S)=L_{i}, \quad U_{i}<L_{i}<N_{i}, \quad i=1,2 .
$$

(iv) Full buffer state probability,

$$
\sum_{S \in Q} f_{i}(S) P(S)=\phi_{i}, \quad 0<\phi_{i}<1, \quad i=1,2,
$$

where $\phi$ satisfies the flow balance equations, namely

$$
\lambda_{i}\left(1-\pi_{i}\right)=\mu_{i} U_{i}, \quad i=1,2 .
$$

The choice of mean values (5)-(8) is based on the type of constraints used for the ME analysis of stable single class FCFS G/G/ $1 / N$ queue [23]. If additional constraints are used, it is no longer feasible to capture a closed form ME solution at the building block level, with clearly, adverse implications on the efficiency of an iterative queue-by-queue decomposition algorithm for general QNMs. Conversely, if one or more constraints form the set (5)-(8) are missing, it is expected that the accuracy of the ME solution will be generally reduced.

\subsection{ME solution}

The form of the state probability distribution, $P(S), S \in Q$, can be characterised by maximising the entropy functional

$$
H(P)=-\sum_{S} P(S) \log P(S)
$$

subject to constraints (5)-(8). By employing Lagrange's method of undetermined multipliers the following solution is obtained:

$$
P(S)=\frac{1}{Z} \prod_{i=1}^{R} x_{i}^{n_{i}(S)} g_{i}^{s_{i}(S)} y_{i}^{f_{i}(S)}, \quad \forall S \in Q
$$

where $Z$ is the normalising constant given by 


$$
Z=\sum_{S \in Q}\left(\prod_{i=1}^{R} x_{i}^{n_{i}(S)} g_{i}^{S_{i}(S)} y_{i}^{f_{i}(S)}\right)
$$

and $x_{i}, g_{i}, y_{i}$ are the Lagrangian coefficients corresponding to constraints (6)-(8), respectively.

\subsection{State probability distribution}

Defining the sets

$$
\begin{aligned}
& S_{0}=\{S / S \in Q: s(S)=0\}, \\
& Q_{k}=\{S \in Q: n(S)=k \& k \geqslant 1\} .
\end{aligned}
$$

It is implied, after some manipulation, that the aggregate ME state probability distribution is given by

$$
\begin{aligned}
& P\left(S_{0}\right)=\frac{1}{Z}, \\
& P(k)=\frac{1}{Z} \sum_{i=1}^{R} x_{i} g_{i} y_{i}^{\delta_{i}(k)} C(k-1), \quad 1 \leqslant k \leqslant N_{2}, \\
& Z=1+\sum_{k=1}^{N_{2}} \sum_{i=1}^{R} x_{i} g_{i} y_{i}^{\delta_{i}(k)} C(k-1),
\end{aligned}
$$

where $i=1,2 R=2 ; \quad \delta_{i}(k)=1$, if $k \geqslant N_{i}$, or 0 , otherwise; and $C(v)=x_{2}^{\delta\left(v+1-N_{1}\right)}\left(x_{1}+x_{2}\right)^{\delta(v)}$, at which $\delta\left(v+1-N_{1}\right)=0$, if $v+1-N_{1} \leqslant 0$, or $v+1-N_{1}$, otherwise; and $\delta(v)=v$, if $v<N_{1}$, or $N_{1}-1$, otherwise.

\subsection{Blocking probability}

The flow balance condition (9), which is used in deriving the Lagrangian coefficient $y_{i}$, is characterised by the blocking probabilities $\pi_{i}$ of a censored GGeo/GGeo/1/\{N $\left.N_{1}, N_{2}\right\}$ queue under AF buffer management policy. These blocking probabilities can be approximated based on a censored GGeo/GGeo/1/N queue [24] as follows:

$$
\pi_{i}=\sum_{k=0}^{N_{2}} \delta_{i}(k)\left(1-\tau_{a i}\right)^{\xi(k)} P(k),
$$

where

$$
\begin{aligned}
& \delta_{i}(k)= \begin{cases}\frac{\tau_{s i}}{\tau_{s i}\left(1-\tau_{a i}\right)+\tau_{a i}}, & i=1 \& k=0, \\
1, & \text { otherwise, }\end{cases} \\
& \xi(k)= \begin{cases}0, & i=2 \& k \leqslant N_{1}, \\
{\left[N_{1}-k\right]^{+},} & \text {otherwise }\end{cases}
\end{aligned}
$$

and $\left[N_{1}-k\right]^{+}=N_{1}-k$, if $k<N_{1}$, or 0 , otherwise. Where $\tau_{a}=2 /\left(1+C_{a}^{2}+\Lambda\right)$, and $\tau_{s}=2 /\left(1+C_{s}^{2}+\mu\right)$, where $C_{a}^{2}$ and $C_{s}^{2}$ are the squared coefficients of variation for the inter-arrival and inter-departure times, respectively.

\subsection{The Lagrangian coefficients $\left\{x_{i}, g_{i}, y_{i}\right\}$}

The Lagrangian coefficients $x_{i}$ and $g_{i}$ can be approximated analytically by making asymptotic connections to an infinite capacity queues. Assuming $x_{i}$ and $g_{i}$ are invariant to the total buffer capacity size $N_{2}$, it can be established that

$$
\begin{gathered}
x_{i}=\frac{<n_{i}>-\rho_{i}}{<n>}, \\
g_{i}=\frac{(1-X) \rho_{i}}{(1-\rho) x_{i}},
\end{gathered}
$$

where $X=\sum_{i=1}^{R} x_{i},\left\langle n>=\sum_{i=1}^{R}<n_{i}>\right.$ and $\left\langle n_{i}\right\rangle$ is the asymptotic marginal mean queue length of a multiple class queue. Note the statistics $\left\langle n_{i}\right\rangle, i=1,2$ can be determined by (cf Kouvatsos et al. [25]) under FCFS rule:

$$
<n_{i}>=\frac{\rho_{i}}{2}\left(C_{a i}^{2}+1\right)+\frac{1}{2(1-\rho)} \sum_{j=1}^{R} \frac{\Lambda_{i}}{\Lambda_{j}} \rho_{j}^{2}\left(C_{a j}^{2}+C_{s j}^{2}\right)
$$

where $\rho=\sum_{i=1}^{R} \rho_{i}$ and $\rho_{i}=\frac{\Lambda_{i}}{\mu_{i}}$

By substituting the value of state probabilities, $P(k), k=0,1, \ldots, \mathrm{N}_{2}$, and the blocking probabilities, $\pi_{i}$, into the flow balance condition (9), the Lagrangian coefficient $y_{i}$, lead to the following expression: 


$$
\begin{aligned}
& y_{i}=\frac{\rho_{1}}{\sum_{v=N_{1}-1}^{N_{2}-1} x_{i} g_{i} C(v)}\left[1+\sum_{j=1}^{R} x_{j} g_{j} \sum_{v=\psi(i)} y_{j}^{\delta_{j}(v)} C(v)-\sum_{j=1}^{R} x_{j} g_{j} \sum_{v=\theta(i)}^{N_{i}-1}\left(1-\tau_{a i}\right)^{N_{i}-v} C(v-1)-\frac{1}{\rho_{i}} x_{i} g_{i} \sum_{v=0}^{N_{i}-2} C(v)-\xi(i)\right] \\
& i=1,2 ; \quad R=2 \text {. }
\end{aligned}
$$

\section{Numerical results}

This section presents typical numerical results to demonstrate the credibility of the proposed analytical solutions, subject to various parameter settings, and validation of results against simulation. It also includes further performance studies. These examples focus on evaluation of the system performance in terms of mean queue length (MQL), throughput, delay, blocking probability and effect of traffic burstiness upon various arrival rates.

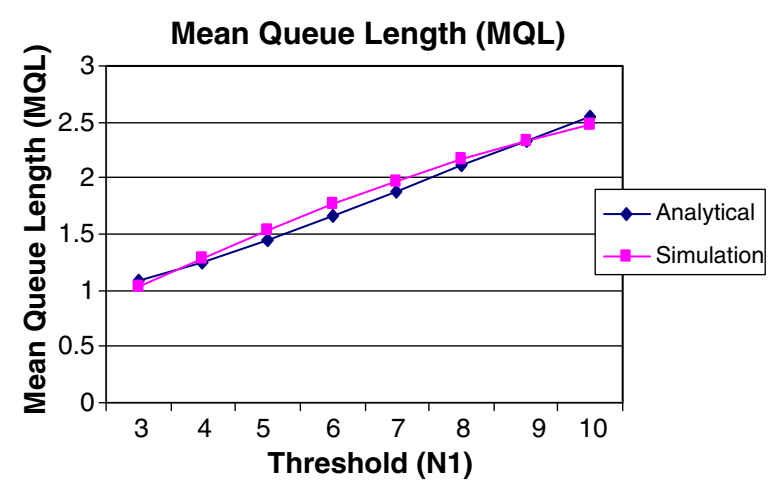

Fig. 3. Results comparison for MQL.

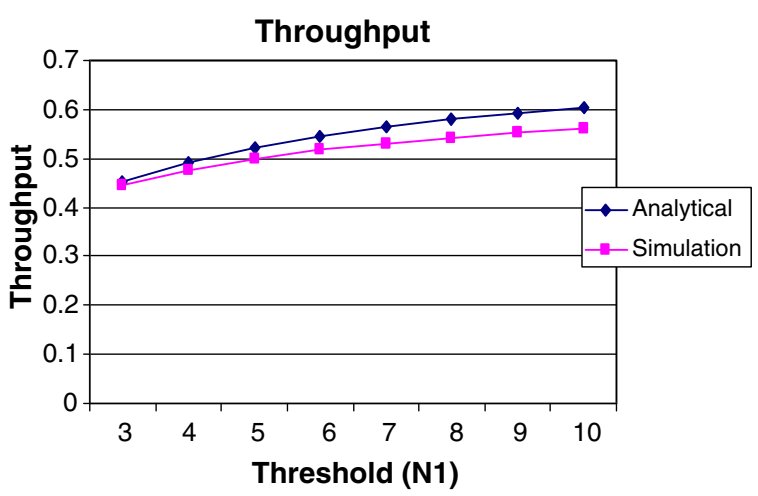

Fig. 4. Results comparison for throughput.

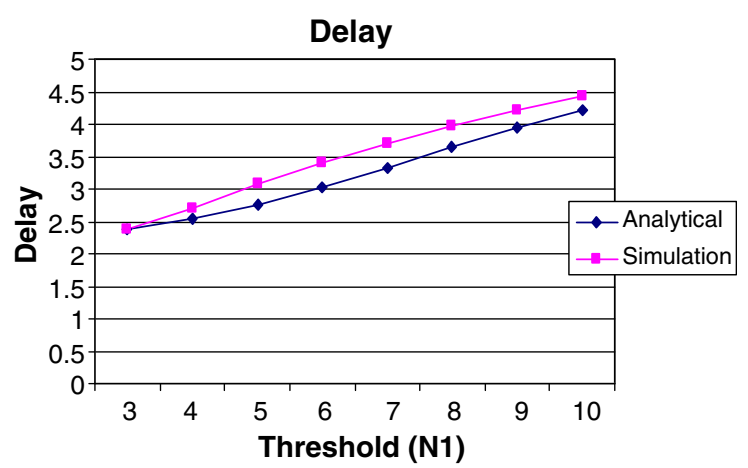

Fig. 5. Results comparison for delay. 


\subsection{Credibility of analytical model}

In this section, the credibility of the proposed analytical model against simulation is examined by means of a comparative study (c.f. Figs. 3-7). A new simulation model has been developed, based on QNAP-2 [26] for a node with queue thresholds and bursty external traffic. It can be observed that, in all experiments, the analytical approximation results are very comparable to the corresponding simulation results.

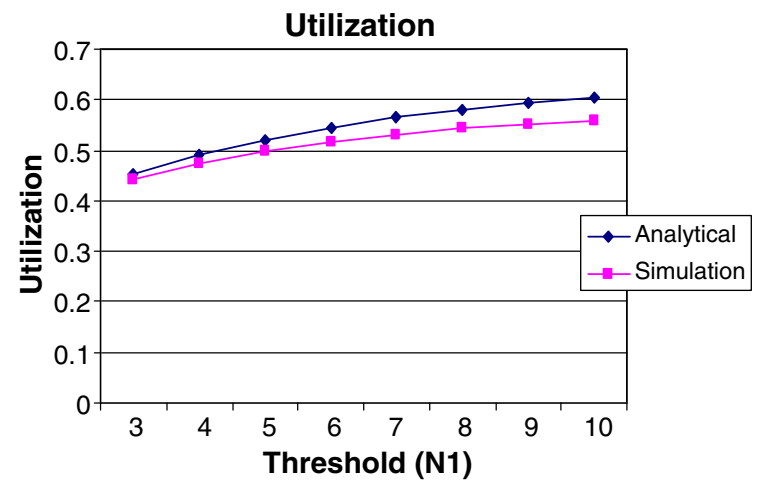

Fig. 6. Results comparison for utilization.

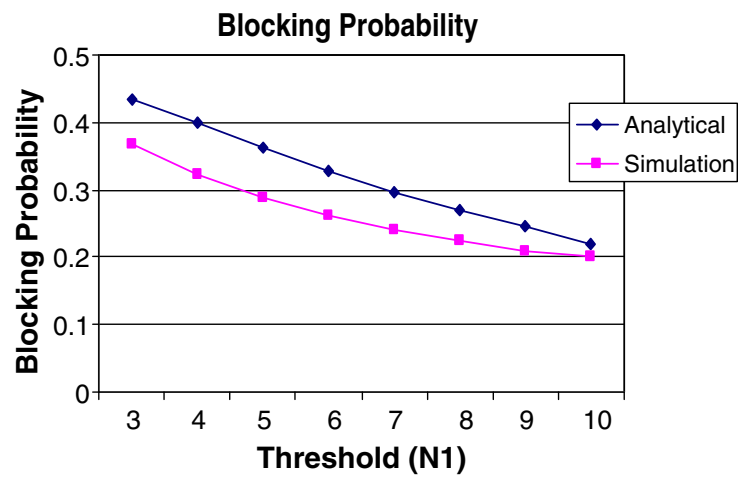

Fig. 7. Results comparison for blocking probability.

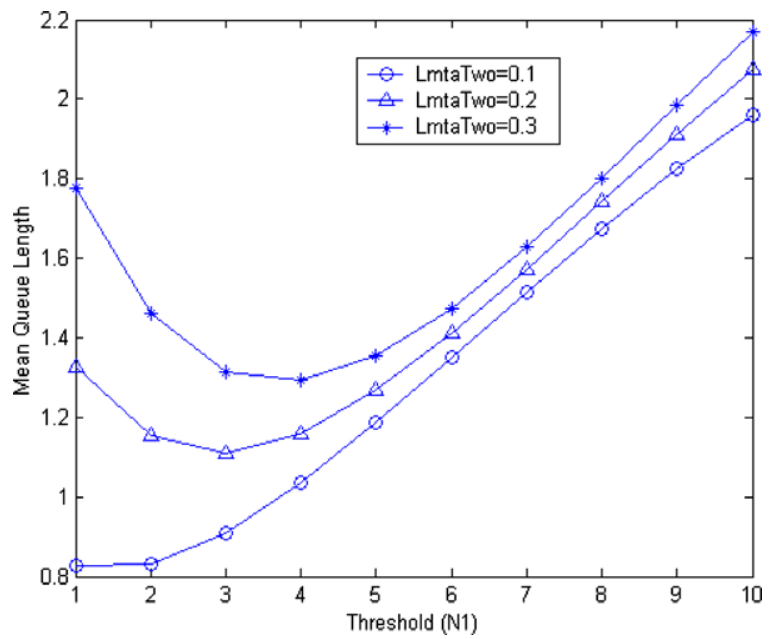

Fig. 8. MQL with different value of $\lambda_{2}$. 
Figs. 3 and 4 show the comparison results of MQL and throughput values for a range of threshold values,respectively. Similarly, Figs. 5-7 demonstrate the comparison results of delay, server utilization and blocking probability for different threshold values against the simulations, respectively. It can be seen that MQL, throughput, delay and server utilization increase as we increase the threshold value, whilst blocking probability decreases. All these examples show performance measures for analytical results and simulation validation comparison with non-varying input parameters as $\lambda_{1}=0.6, \lambda_{2}=0.1, \mu=1.0$, $\mathrm{Ca}=\mathrm{Cs}=3$ for the total system capacity $N_{2}=10$ whilst $N_{1}$ varies over a given range.

\subsection{Performance study of the analytical model}

This section presents the performance study of the proposed analytical model. These results focus on evaluation of the system performance in terms of MQL, throughput, delay, blocking probability and the effect of traffic burstiness upon various arrival rates.

Figs. 8-10 show the comparison results of MQL, utilization and blocking probability over different values of arrival rate $\lambda_{2}$, respectively, when the other input parameter values remain fixed, such as $\lambda_{1}=0.6, \mu=1.2, \mathrm{Ca}=\mathrm{Cs}=3$, the total system capacity $N_{2}=10$ and the threshold $N_{1}$ varies over a given range. Figs. 8 and 9 indicate that the values of MQL and system utilization are higher for the higher arrival rate $\lambda_{2}$ (e.g. $\lambda_{2}=0.3$ ) and they also exhibit the same behaviour for the higher threshold settings based on the same arrival rate $\lambda_{2}$. Note that the system behaves normally for the threshold value greater than or equal to the traffic bursty parameter Ca. However, for small values of Ca (e.g. $N_{1}<\mathrm{Ca}$ ), the queue does not behave normally since there is not enough space to accommodate an arriving burst of traffic. Fig. 10 shows the value of blocking probability is lower

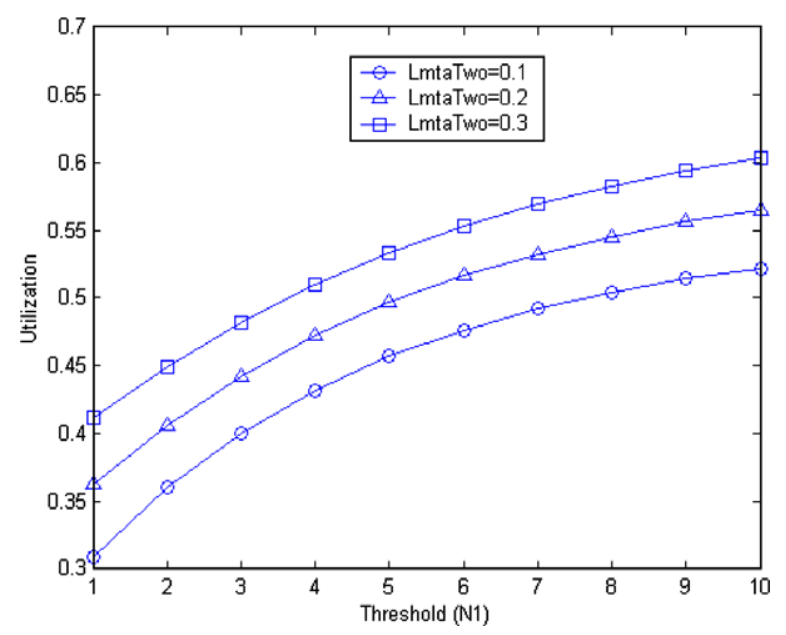

Fig. 9. Utilization with different value of $\lambda_{2}$.

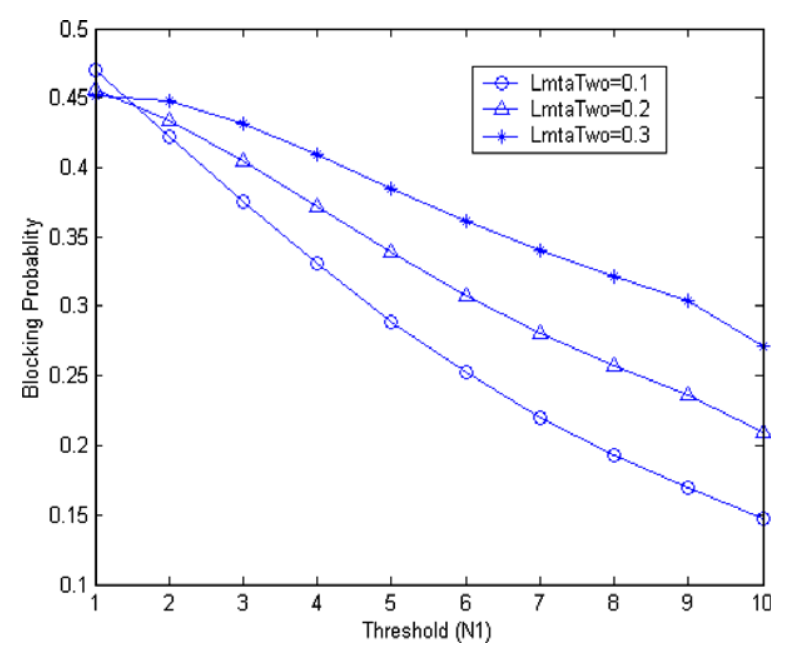

Fig. 10. Blocking probability with different value of $\lambda_{2}$. 


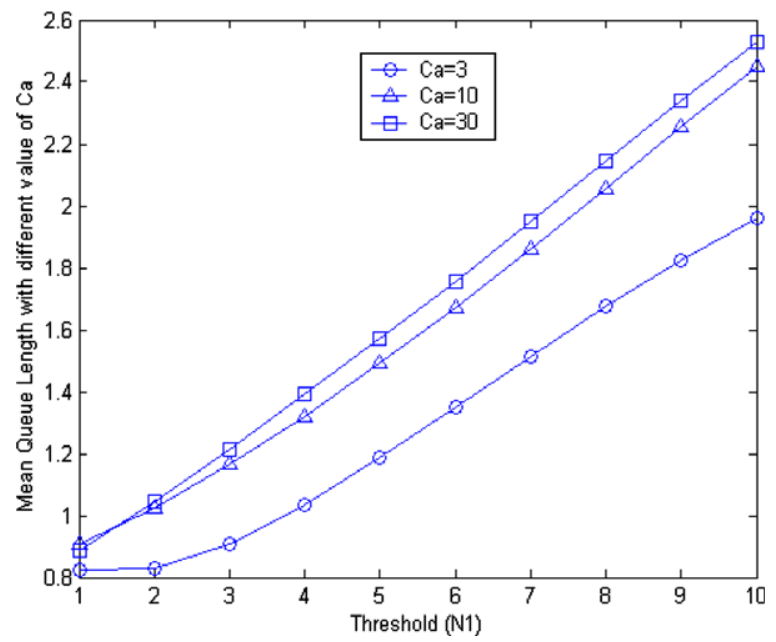

Fig. 11. MQL with different value of squared coefficient variation of the inter-arrival time (Ca).

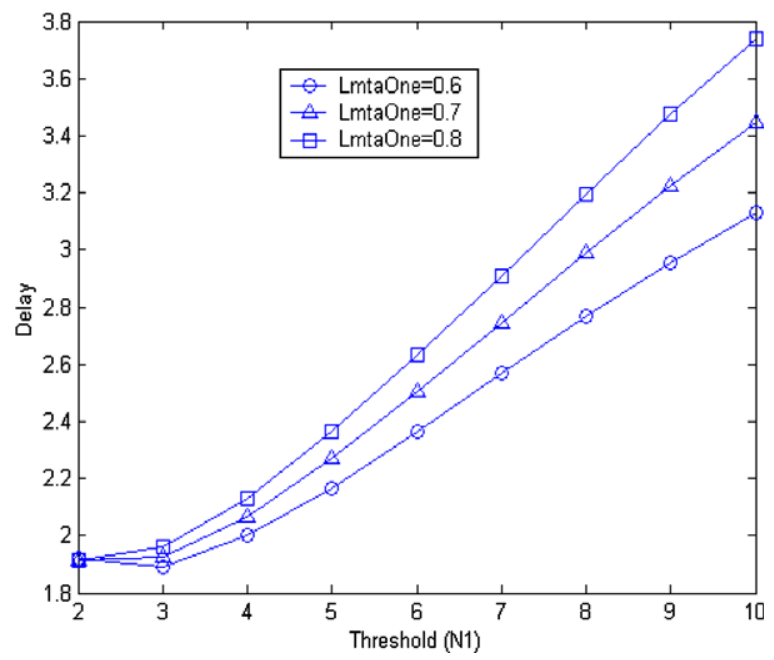

Fig. 12. Delay with different value of $\lambda_{1}$.

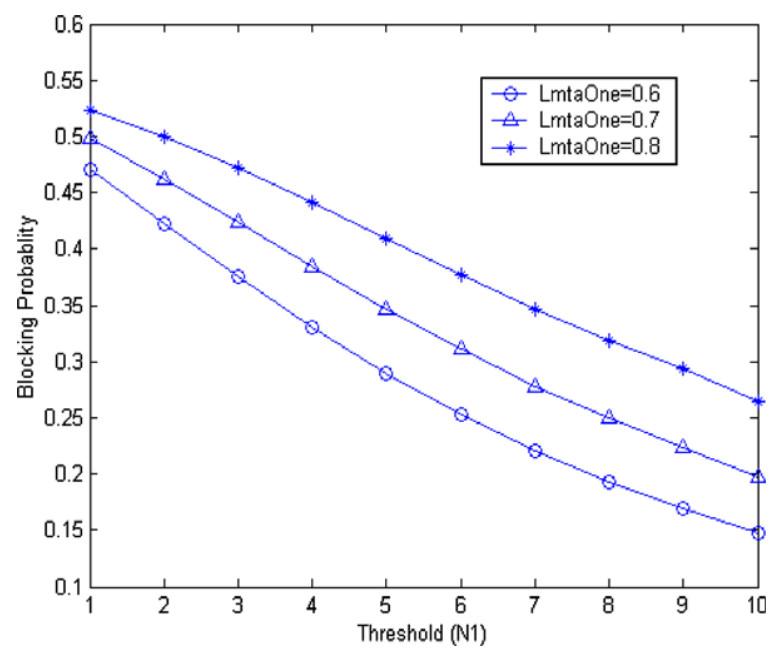

Fig. 13. Blocking probability with different value of $\lambda_{1}$. 
for the lower arrival rate $\lambda_{2}$ (e.g. $\lambda_{2}=0.1$ ) and based on the same arrival rate, blocking probability decreases following the threshold value increasing.

Fig. 11 demonstrates the effect of traffic burstiness upon MQL using various values of the squared coefficient of variation of the inter-arrival time (Ca). It is shown that the higher burstiness traffic causes higher system mean queue length for the same threshold settings based on the assumption that the total amount of the traffic remains constant.

Figs. 12 and 13 show the comparison results of delay and blocking probability over different values of arrival rate $\lambda_{1}$, respectively, when the other input parameter values are fixed, such as $\lambda_{2}=0.1, \mu=1.2, \mathrm{Ca}=\mathrm{Cs}=3$, the system total capacity $N_{2}=10$ and $N_{1}$ varies over a given range. Figs. 12 and 13 indicate the values of delay and blocking probability are higher for the higher arrival rate $\lambda_{1}$ (e.g. $\lambda_{1}=0.8$ ) and based on the same arrival rate, delay increases as we increase the values of threshold settings. However, blocking probability decreases as the threshold value increases.

\section{Conclusions and future work}

An analytical model, based on the principle of maximum entropy, has been presented to evaluate the performance of a congestion control mechanism for implementing the AQM scheme. In this context, a stable discrete-time single server GGeo/ GGeo/1/\{ $\left.N_{1}, N_{2}\right\}$ censored queue finite capacity queue with $N_{1}$ threshold value, $N_{2}$ total buffer capacity, FCFS scheduling discipline and arrival first (AF) buffer management policy has been analysed. Closed form analytical expressions for various performance metrics including state and blocking probabilities have been presented. The traffic source slows down the arrival process as soon as the number of packets in the queue reaches the threshold and jobs are blocked once the queue becomes full. Different QoS requirements under various load conditions can be satisfied by adjusting the threshold value. Typical numerical results were included to demonstrate the credibility of the proposed ME solution against simulation results. The system can be potentially used as a model for congestion control based on the RED mechanism. Future work will focus on implementation of this algorithm for various types of network topology.

\section{Acknowledgement}

This work was sponsored by the Engineering and Physical Science Research Council (EPSRC), UK, under Grant GR/S01658/ 01 .

\section{References}

[1] Jian-Min Li, Indra Widjaja, Marcel F. Neuts, Congestion detection in ATM network, Performance Evaluation 34 (1998) 147-168.

[2] I.U. Awan, L Guan, X.G. Wang, Analysis of discrete time queueing networks under buffer threshold scheme, in: International Workshop on Performance Modelling and Evaluation in Computer and Telecommunication Networks (PMECT07) in Conjunction with 16th International Conference on Computer Communications and Networks (ICCCN2007), IEEE Communications Society, Hawaii, USA, ISBN: 1-4244-1251-X, August 2007.

[3] B. Braden et al., Recommendations on Queue Management and Congestion Avoidance in the Internet, IETF RFC 2309 , April 1998.

[4] S. Floyd, V. Jacobson, Random early detection gateways for congestion avoidance, IEEE/ACM Transaction on Networking 1 (4) (1993) $397-413$.

[5] D. Lapsley, S. Low, Ransom early marking for internet congestion control, in: Proceeding of GlobeCom'99, December 1999, pp. $1747-1752$.

[6] S. Athuraliya, D. Lapsley, S. Low, An Enhanced Random Early Marking Algorithm for Internet Flow Control, Infocom, 2000.

[7] R. Gibbens, F. Kelly, Distributed connection acceptance control for a connectionless network, in: Proceeding of the 16th Intl. Teletraffic Congress, Edinburgh, Scotland, June 1999.

[8] S. Kunniyur, R. Srikant, End-to-End congestion control: utility function, random losses and ECN marks, in: Proceedings of Infocom 2000, Tel Aviv, Israel, March 2000.

[9] S. Kunniyur, R. Srikant, A time-scale decomposition approach to adaptive ECN marking, in: Proceeding of Infocom 2001, Alaska, Anchorage, April 2001.

[10] C. Hoolot, V. Misra, D. Towlsey, W. Gong, On designing improved controllers for AQM routers supporting TCP flows, UMass CMPSCI Technical Report $00-42,2000$.

[11] H. Alazemi, A. Mokhtar, M. Azizoglu, Stochastic modelling of random early detection gateway in tcp networks, in: IEEE Global Telecommunications Conference, GLOBECOM'00, vol. 3, 2000, pp. 1747-1751.

[12] M. May, T. Bonald, J. Bolot, Analytical evaluation of RED Prformance, in: Proceedings of INFOCOM’ 2000, 2000.

[13] A. Mokhtar, M. Azizoglu, A random early discard frame work for congestion control in ATM networks, in: IEEE ATM Workshop'99 Proceedings, Tokyo, Japan, 1999, pp. 45-50.

[14] H. Alazemi, A. Mokhtar, M. Azizoglu, Stochastic approach for modelling random early detection gateways in TCP/IP networks, IEEE International Conference on Communications 8 (2001) 2385-2390.

[15] E.T. Jaynes, Information Theory and Statistical Mechanics, Physical Review 106 (1957) 620-630.

[16] E.T. Jaynes, Information theory and statistical mechanics, II, Physical Review 108 (1957) 171-190.

[17] J.E. Shore, R.W. Johnson, Axiomatic derivation of the principle of maximum entropy and the principle of minimum-cross entropy, IEEE Transactions of Information Theory 26 (1980) 26-37.

[18] M. Tribus, Rational Descriptions, Decisions, and Designs, Pergamon Press, New York, 1969.

[19] D.D. Kouvatsos, Entropy maximisation and queueing network models, Annals of Operation Research 48 (1994) 63-126.

[20] D.D. Kouvatsos, N.M. Tabet-Aouel, GGeo-type approximations for general discrete-time queueing systems, in: H.G. Perros et al. (ed.), Proc. of IFIP Workshop on Modelling and Performance Evaluation of ATM Technology, La Martinique, January 1993.

[21] D.D. Kouvatsos, N.M. Tabet-Aouel, S.G. Denazis, ME-based approximations for general discrete-time queueing models, Journal of Performance Evaluation 21 (1994) 81-109.

[22] D.D. Kouvatsos, Irfan U. Awan, R.J. Fretwell, G. Dimakopoulos, A cost-effective approximation for SRD traffic in arbitrary multi-buffered networks, Journal of Computer Networks 34 (2000) 97-113.

[23] D. Kouvatsos, Maximum entropy and the G/G/1/N queue, Acta Informatica 23 (1986) 545-565.

[24] D.D. Kouvatsos, N.M. Tabet-Aouel, S.G. Denazis, Approximation analysis of discrete-time networks with or without blocking, in: H.G. Perros, Y. Viniotis (Eds.), High Speed Networks and their Performance (C-21), 1994. 
[25] D.D. Kouvatsos, P.H. Georgatsos, N.M. Tabet-Aouel, A universal maximum entropy algorithm for general multiple class open networks with mixed service disciplines, in: R. Puigjaner, D. Potier (Eds.), Modelling Techniques and Tools for Computer Performance Evaluation, Plenum, 1989, pp. 397419.

[26] M. Veran, D. Potier, QNAP-2: a portable environment for queueing network modelling techniques and tools for performance analysis, in: D. Potier (Ed.), North-Holland, 1985.

[27] D.D. Kouvatsos, I. Awan, MEM for arbitrary closed queuing networks with RS-blocking and multiple job classes, Annals of Operations Research 79 (1998) 231-269 (special issue on queuing networks with finite capacities).

[28] D. Kouvatsos, I. Awan, Analytical approximation of arbitrary queueing networks with blocking and priorities, Journal of Performance Evaluation 51 (2-4) (2005) 191-227. 